\title{
Aproximación al estado de salud de la comunidad indígena Yanacona en la ciudad de Bogotá, D.C., por medio de pruebas de laboratorio clínico.
}

\author{
Martha Leonor Castillo Bohórquez ${ }^{1}$, Ana lucia oliveros ${ }^{1}$, Ana Isabel Mora \\ Bautista $^{1}$, Erika Cediel ${ }^{2}$, Marcela Morales ${ }^{2}$. \\ 1. Docentes investigadoras Semillero ERITRON Universidad Colegio Mayor de Cundinamarca. \\ 2. Estudiantes del programa de Bacteriología y Laboratorio Clínico de la Universidad \\ Colegio Mayor de Cundinamarca.
}

Correspondencia: mlcastillo@unicolmayor.edu.co

Recibido: 19/07/2012 Aceptado: 23/11/2012

\begin{abstract}
Resumen
El estudio se realizó con la comunidad indígena Yanacona, nativos del macizo Colombiano que actualmente están ubicados en la ciudad de Bogotá; en el barrio San Joaquín de la localidad de Ciudad Bolívar. Esta investigación siguió una metodología descriptiva, transversal, se estudiaron 21 integrantes pertenecientes a la comunidad; hombres y mujeres entre 19 a 48 años de edad, a los que se realizaron tres pruebas de laboratorio clínico de rutina: uroanálisis, coprológico y hemograma. Como prueba especial se realizó la ferritina sérica como medida indirecta de los depósitos de hierro para verificar una anemia ferropénica. Basados en los resultados de los exámenes se observó en bajo porcentaje infecciones urinarias y parasitosis, ninguno presento anemia. Estos hallazgos sugieren una población adaptada a las condiciones de vida de la ciudad pero que requieren actividades de promoción de salud y prevención de la enfermedad.
\end{abstract}

Palabras clave: Indígenas, salud, factores socioculturales, pruebas laboratorio.

\section{Approach to Yanacona indigenous community health status in Bogotá, DC through clinical laboratory tests}

\begin{abstract}
Abstrac
This study was carried out within the indigenous community Yanacona, natives of Colombian Massif which were situated in Bogota city (San Joaquin- Ciudad Bolivar). A cross-sectional descriptive study was performed studying 21 members from the community, men and women between 19-48 years old. Three routine clinical laboratory tests were carried out: urinalysis, stool analysis and blood count. To check for iron-deficiency anemia a serum ferritin test was performed as indirect measurement of iron deposits. According with the results a low percentage of urinary and parasitic infections was observed and no cases of anemia. These findings indicated a population adapted to the city life conditions but requiring health promotion activities and disease prevention. Keywords: Indigenous, health, sociocultural factors, laboratory tests.
\end{abstract}




\section{Introducción}

En la actualidad no se cuenta con la suficiente evidencia documental y existe escasa información sobre estadísticas e indicadores de salud de las comunidades indígenas especialmente de aquellas que son desplazadas a las ciudades por diferentes razones. Con el presente informe, se pretende adquirir información de una comunidad específica por medio de un sondeo diagnostico que proporcione un panorama general de su estado de salud.

En Colombia, la disminución de poblaciones indígenas se ve condicionada por diferentes factores; violencia por el conflicto del armado del país, y particularmente, por problemas en el acceso a servicios de salud. Son pocas las comunidades indígenas que cuentan con la información, apoyo y seguimiento por parte del Estado. Por otra parte, la mayoría de estas poblaciones prefieren ser atendidas por sus médicos ancestrales ya que confían más en el saber tradicional.

Así mismo, la información epidemiológica acerca de la salud en comunidades indígenas es muy limitada, las Organizaciones como la OMS cuentan con cifras estimadas sobre la población aborigen mundial calculada en 370 millones de indígenas (1). En Colombia, según el DANE, la población indígena representa el 3,4\% de los habitantes totales; distribuidos en 84 etnias (2) por todo el país. La escasa información se debe a que son pocos los proyectos que llegan a ser concluidos o que generan un verdadero impacto en la población indígena.

Para este estudio, se escogió la comunidad indígena Yanacona con el fin de obtener información de las condiciones y hábitos de vida adquiridos en la ciudad de Bogotá. Las pruebas que se realizaron fueron exámenes clínicos de rutina. La correlación del cuadro hemático, el uroanálisis y el coprológico permitieron analizar el estado básico de los individuos en estudio. Como prueba especializada se realizó la ferritina sérica para encaminar las alteraciones que puedan ser encontradas en el cuadro rojo hacia una anemia ferropénica.

\section{Materiales y métodos}

Exámenes de laboratorio. A las muestras obtenidas por punción venosa con EDTA se les realizo el cuadro hemático automatizado, para determinar anormalidades en las células sanguíneas y presencia de anemia. El equipo utilizado fue el MINDRAY BC-3000 PLUS, de tercera generación de la Universidad Colegio Mayor de Cundinamarca.

Como prueba especializada se realizó la Ferritina sérica para evidenciar las deficiencias de hierro, asociado a la anemia ferropénica por hábitos alimenticios. La técnica utilizada fue enzimoinmunoanálisis (ELISA). Se utilizó: Human ferritin enzyme immunoassay test kit de Diagnostic Automation, INC.

Se recolectaron muestras de orina para uroanálisis, con el fin de determinar; infecciones urinarias, anormalidades del metabolismo o daño renal. Se realizó examen físico, químico y microscópico. Para examen químico se utilizaron las tiras reactivas URINE STRIP 10 de la casa comercial WIENER.

Por último, se recolectaron muestras de materia fecal para coprológico y determinar la presencia de parásitos. Se realizó examen físico - químico y examen microscópico.

Análisis estadístico. Se analizó la distribución de las variables de razón, aplicando la prueba de ShapiroWilk de normalidad. Las variables de razón (edad, $\mathrm{pH}$ y cuadro hemático) presentaron una distribución normal, por lo tanto, se describen a través de medidas de agrupación (promedio) y dispersión (desviación estándar (SD)). Las variables categóricas se describen con proporciones. Los datos del cuadro hemático se describen adicionalmente según los parámetros clínicos. Se construyeron tablas de 2X2 y NXM para establecer la asociación entre variables de exposición con las variables de enfermedad, infección urinaria y parasitosis. La prueba de Fisher se utilizó para verificar la asociación cuando los datos esperados en las tablas de $2 \mathrm{X} 2$ son menores de 5; la significancia estadística se definio con una $p \leq 0,05$. Se utilizó el programa estadístico SPSS versión 17,0. 


\section{Resultados}

En la capital residen unas 79 familias indígenas, aproximadamente unas 200 personas que habitan en las localidades de Rafael Uribe, Bosa, Suba y Ciudad Bolívar (3). Para el presente estudio se contó con el permiso del Gobernador de la comunidad para trabajar solo con la población de la localidad de Ciudad Bolívar en el barrio San Joaquín, que aproximadamente reúne 40 personas mayores de edad, de estas 21 personas aceptaron participar en el estudio, asistieron el día de la toma de muestra y se les aplicó la encuesta. La población se componía en un $52,4 \%$ en hombres y un $47,6 \%$ en mujeres. El promedio de edad fue de 31 años. Otras características socio demográficos se presentan en la tabla 1 .

Tabla 1. Características socio demográficas de la comunidad indígena Yanacona, ubicados en la ciudad de Bogotá D.C., en el barrio San Joaquín de la localidad de Ciudad Bolívar.

\begin{tabular}{|c|c|c|c|}
\hline \multicolumn{2}{|l|}{ Característica } & $\begin{array}{c}\text { No } \\
\text { personas }\end{array}$ & Porcentaje \\
\hline \multirow{4}{*}{ Estado civil } & Soltero & 7 & $33,3 \%$ \\
\hline & Unión libre & 8 & $38,1 \%$ \\
\hline & Casado & 6 & $28,6 \%$ \\
\hline & Total & 21 & \\
\hline \multirow{3}{*}{$\begin{array}{l}\text { Estrato } \\
\text { socioeconómico }\end{array}$} & 1 & 18 & $85,7 \%$ \\
\hline & 2 & 3 & $14,3 \%$ \\
\hline & Total & 21 & \\
\hline \multirow{11}{*}{ Ocupación } & Ama de casa & 6 & $28,6 \%$ \\
\hline & Oficios varios & 4 & $19,0 \%$ \\
\hline & Operario & 3 & $14,3 \%$ \\
\hline & $\begin{array}{l}\text { Supervisor } \\
\text { calidad }\end{array}$ & 1 & $4,8 \%$ \\
\hline & Estudiante & 2 & $9,5 \%$ \\
\hline & Secretaria & 1 & $4,8 \%$ \\
\hline & Conductor & 1 & $4,8 \%$ \\
\hline & Militar & 1 & $4,8 \%$ \\
\hline & $\begin{array}{l}\text { Trabajador } \\
\text { social }\end{array}$ & 1 & $4,8 \%$ \\
\hline & Ninguno & 1 & $4,8 \%$ \\
\hline & Total & 21 & \\
\hline
\end{tabular}

Por medio de la encuesta se indagó acerca de la razón por la cual se encuentran asentados en la ciudad de Bogotá y no en su tierra nativa, en el Departamento del Cauca; el 52,4\% llegó a la ciudad en busca de oportunidades laborales, con un tiempo de residencia de 5 a 10 años aproximadamente. Por el tiempo que llevan viviendo en la ciudad, sus patrones culturales han cambiado y han sido forzados a acoplarse a las condiciones de vida urbanas.

Para el estudio fue importante resaltar las condiciones, hábitos de higiene y aseo dentro de la vivienda. La totalidad de personas cuentan con todos los servicios públicos básicos. En cuanto a los hábitos alimenticios el 71,4\% como mínimo consumen 3 comidas diarias. La mayoría lava sus alimentos antes de ser consumidos, hierve el agua que usa para prepararlos y el agua para consumo.

En la población indígena se encontró que la mayoría están afiliados al sistema de seguridad social, y pertenecen al régimen contributivo, ya que son trabajadores. Sólo el 23,8\% de la comunidad refiere que por el hecho de ser indígena se ha sentido discriminada en la atención de salud.

Por el tipo de comunidad surge el interrogante ¿Qué tipo de medicina utilizan con más frecuencia?, el resultado obtenido fue que la mayoría utiliza la medicina tradicional como se presenta en la tabla 2.

Tabla 2. Medicina que utilizan los indígena Yanacona, ubicados en la ciudad de Bogotá, D.C.

\begin{tabular}{llcc} 
& Gripa & 3 & 14,29 \\
& Dolor & 9 & 42,86 \\
$\begin{array}{l}\text { Ocasiones } \\
\text { medicina } \\
\text { tradicional }\end{array}$ & Gripa y dolor & 3 & 14,29 \\
& Curaciones y & 1 & 4,76 \\
& prevención & 5 & 23,81 \\
& Ninguno & 21 & 100,00 \\
& Total & 3 & 14,29 \\
& Infecciones & 3 & 14,29 \\
& Accidentes & 1 & 4,76 \\
& Controles médicos & 1 & 4,76 \\
& Exámenes rutinarios & 1 & 4,76 \\
Ocasiones & Dolor & 1 & 4,76 \\
medicina & Odontología & 1 & 4,76 \\
& Todos los casos & 1 & 4,76 \\
& Fracturas & 1 & 4,76 \\
& Parto & 1 & 38,10 \\
& Nunca & 8 & 100,00 \\
\hline
\end{tabular}




\section{Estado de salud}

Los resultados del uroanálisis se pueden observar en la tabla 3, de los cuales dos personas presentaron infección urinaria.

Tabla 3. Resultados del uroanálisis de la comunidad indígena Yanacona, ubicados en la ciudad de Bogotá, D.C.

\begin{tabular}{|c|c|c|c|}
\hline $\begin{array}{l}\text { Característica } \\
\text { del uroanálisis }\end{array}$ & & No & Porcentaje \\
\hline \multirow{3}{*}{ Nitritos } & Negativo & 19 & 90,5 \\
\hline & Positivo & 2 & 9,5 \\
\hline & Total & 21 & \\
\hline \multirow{6}{*}{ Leucocitos } & Negativo & 15 & 71,4 \\
\hline & Trazas & 3 & 14,3 \\
\hline & 10 a $23+$ & 1 & 4,8 \\
\hline & $75++$ & 1 & 4,8 \\
\hline & +++ & 1 & 4,8 \\
\hline & Total & 21 & \\
\hline \multirow{11}{*}{$\begin{array}{l}\text { Leucocitos } \\
\text { microscópicos }\end{array}$} & Ocasionales & 4 & 19,0 \\
\hline & $0-1$ & 4 & 19,0 \\
\hline & $0-2$ & 3 & 14,3 \\
\hline & $1-3$ & 2 & 9,5 \\
\hline & $2-4$ & 1 & 4,8 \\
\hline & $3-5$ & 2 & 9,5 \\
\hline & $5-7$ & 1 & 4,8 \\
\hline & $5-10$ & 1 & 4,8 \\
\hline & $8-10$ & 2 & 9,5 \\
\hline & Incontables & 1 & 4,8 \\
\hline & Total & 21 & \\
\hline \multirow{5}{*}{ Bacterias } & Escasas & 8 & 38,1 \\
\hline & + & 9 & 42,9 \\
\hline & ++ & 2 & 9,5 \\
\hline & +++ & 2 & 9,5 \\
\hline & Total & 21 & \\
\hline \multirow{4}{*}{$\begin{array}{l}\text { Infección } \\
\text { urinaria }\end{array}$} & No infección & 16 & 76,2 \\
\hline & Infección & 2 & 9,5 \\
\hline & Contaminada & 3 & 14,3 \\
\hline & Total & 21 & \\
\hline
\end{tabular}

En cuanto al cuadro hemático, la mayoría de la población presenta valores dentro del rango de normalidad, sin alteraciones dentro del cuadro blanco ni rojo. Tampoco se encontró presencia de anemia.
En el examen coprológico se pudo evidenciar que 5 personas de la comunidad tienen parasitosis intestinal. Los parásitos encontrados se pueden observar en la tabla 4 .

En cuanto a la prueba especial, la ferritina sérica, ningún participante presento valores disminuidos.

Tabla 4. Parásitos encontrados en materia fecal de la comunidad indígena Yanacona, ubicados en la ciudad de Bogotá, D.C.

\begin{tabular}{|c|c|c|c|}
\hline & $\begin{array}{c}\text { Tipo de parasito } \\
\text { aislado }\end{array}$ & $\begin{array}{c}\text { Número de } \\
\text { personas }\end{array}$ & $\begin{array}{l}\text { Porcen- } \\
\text { taje }\end{array}$ \\
\hline \multirow{4}{*}{ Parásitos } & $\begin{array}{l}\text { Quistes complejo } \\
\text { Entamoeba histolyti- } \\
\text { calEntamoeba dispar }\end{array}$ & 3 & $60.0 \%$ \\
\hline & $\begin{array}{l}\text { Blastocistis hominis } \\
\text { y Quistes complejo } \\
\text { Entamoeba histolyti- } \\
\text { calEntamoeba dispar }\end{array}$ & 1 & $20.0 \%$ \\
\hline & $\begin{array}{l}\text { Quistes Giardia } \\
\text { lamblia y quistes } \\
\text { Entamoeba coli }\end{array}$ & 1 & $20.0 \%$ \\
\hline & Total & 5 & \\
\hline \multirow{3}{*}{ Parasitosis } & Sin parasitosis & 7 & $58.3 \%$ \\
\hline & Con parasitosis & 5 & $41.7 \%$ \\
\hline & Total & 12 & \\
\hline
\end{tabular}

\section{Discusión}

El presente estudio reveló que la comunidad Yanacona es una población adaptada al estilo de vida de la ciudad de Bogotá, D.C., debido al tiempo de permanencia y a la necesidad de adaptación. Según Colleen, cuando hay desplazamiento indígena se pierde la antigua manera de vivir por la necesidad de asimilar al ambiente nuevo, tener éxito y conseguir trabajo (4). Es el caso de la mayoría de la población Yanacona que se desplazó a ciudad de Bogotá por razones laborales.

La comunidad Yanacona en la ciudad de Bogotá, D.C, a pesar de perder algunos de sus patrones culturales aún conservan su medicina tradicional, lo que no garantiza que su estado de salud sea el mejor. Es lo que señala la OMS, con respecto a la eficacia y seguridad de estas prácticas, 
asegurando que a pesar de la existencia de datos demostrativos de la capacidad para tratar determinadas enfermedades, son escasos los ensayos científicos que evalúen estos procesos (5). Sin embargo, este grupo de personas tienden a mezclar su medicina ancestral con la occidental lo que reduce el número de posibles patologías y complicaciones de enfermedades.

Las infecciones urinarias señaladas en este estudio son presuntivas, ya que se requiere de un urocultivo para confirmarlas. Los casos presentados son pocos y en su totalidad por parte de la población femenina. Como lo indica Arinzon et al. (6) Las infecciones urinarias en mujeres son las infecciones bacterianas más comunes. Más del $50 \%$ de las mujeres han tenido al menos una infección urinaria durante su vida, y del $20 \%$ al $30 \%$ han tenido episodios recurrentes. Además, las infecciones urinarias sin complicaciones son frecuentes en mujeres inmunocompetentes y con una fisiología anatómica normal (6). Otros aspectos asociados al riesgo de tener una infección urinaria son el hecho de ser mujeres jóvenes y casadas. Similar a esto Arizon (6) en su estudio observó una correlación significativa entre la edad y la actividad sexual. La mayoría de sus pacientes que presentaron episodios de infección estaban casadas y tenían una actividad sexual regular.

En lo referente al parasitismo intestinal, en términos de proporción se presenta tanto en hombres como en mujeres. Dicha situación es similar al estudio publicado por Rivero Z, et al (7), donde a pesar que los hombres presentaron un alto porcentaje de parasitismo, no se pudo establecer una diferencia significativa entre los dos géneros (7). Igualmente Devera, et al (8) expone que en un estudio de prevalencia de parasitosis de indígenas de Venezuela no hubo distinción por el género ni la edad (8).

Entre los factores de riesgo que están asociados a la parasitosis se incluyen; los hábitos de higiene y fuente de agua. Según el estudio denominado la
Gran Expedición Humana realizado por la Universidad Javeriana de la ciudad de Bogotá, el parasitismo intestinal en las poblaciones varía según la fuente de agua que utilicen, incidiendo más en aquellas que usan agua de lluvia, río o pozos donde el poliparasitismo es cuatro veces mayor (9). Por la ubicación nativa de estas poblaciones es común la utilización de este tipo de aguas, contrario a los Yanaconas que cuentan con servicio de alcantarillado lo que disminuye el riesgo y en consecuencia el número de casos encontrados.

Sin embargo, en el estudio de la Gran Expedición Humana encontraron que incluso en las comunidades donde realizaban un adecuado manejo de excretas, el parasitismo alcanzo cifras muy altas. Lo que coincide con el presente estudio donde los participantes que aseguran hervir el agua y lavar las frutas y verduras presentan parásitos. Lo anterior sugiere que es indispensable un proceso educativo sobre hábitos de higiene.

Los parásitos encontrados: Blastocistis hominis, Complejo Entamoeba hystolitical Entamoeba dispar, quistes de Giardia intestinalis y quistes de Entamoeba coli, pertenecientes al grupo de protozoarios, predominaron en la comunidad Yanacona, lo que coincide con los datos obtenidos por Devera (8) que encontró prevalencia por Blastocistis hominis y Entamoeba coli en la población Yakariyene de Venezuela, y con Rivero Z, et al, (7) que encontró las mismas especies más el Complejo Entamoebahystolitical Entamoeba dispar en la comunidad Japrería de Venezuela. Es importante resaltar que el porcentaje de parasitismo en estas poblaciones fue mayor debido a que son poblaciones nativas que viven en la zona rural de sus países, con condiciones ambientales y culturales diferentes de los Yanacona que viven en la zona urbana con mejores condiciones de vida.

Referente a la anemia ferropénica, interés de estudio, no se encontraron personas que cursaran con esta patología, debido a que cuentan con 
una buena nutrición y una dieta adecuada de hierro. En contraste, la población indígena Piaroa estudiada por García et al encontró que el $89,6 \%$ de la población presentaba anemia lo que indica un problema de salud y desnutrición importante (10-11).

Para evaluar mejor este aspecto, se realizó la prueba de ferritina sérica, pues como indica Cook, esta prueba demuestra superioridad sobre otras medidas relacionadas con el hierro para la identificación de anemia ferropénica, sin olvidar que esta prueba tiene sus limitaciones en personas con infecciones o inflamaciones (12). Solo una persona tuvo el valor disminuido, lo cual no indica que curse con anemia. Esto basado en Cook, que demuestra que esta prueba detecta la más leve deficiencia de hierro en ausencia de anemia. Además como lo sugiere la OMS se debe relacionar con los datos de la hemoglobina y el hematocrito (13), y en general el cuadro hemático de esta persona fue normal.

De la misma forma, esto sucedió en el estudio de García, et al (14) realizado en una comunidad indígena venezolana donde se vio anormalidad en esta proteína pero no existía anemia, esto fue atribuido a la presencia de diferentes infecciones e inflamaciones (15).

\section{Conclusión}

La población Yanacona ubicada en el barrio San Joaquín en la localidad de ciudad Bolívar de Bogotá, es una población minoritaria, homogénea y aparentemente sana por su ubicación urbana y condiciones de vivienda, en comparación con poblaciones indígenas asentadas en su territorio nativo.

A pesar de sacrificar algunas creencias ancestrales, se evidencia que el traslado a la ciudad mejora el acceso a los servicios de salud y un cambio favorable en sus hábitos de higiene.

\section{Referencias}

1. Organización mundial de la salud. La salud de los pueblos indígenas. [Sitio en internet]. Disponible en: http://www.who. int/mediacentre/factsheets/fs326/es/index.html.Consultado: 5 de marzo de 2011

2. Departamento administrativo nacional de estadística DANE. Colombia una nación multicultural, su diversidad étnica. [Sitio en internet]. Disponible en: http://www.dane.gov.co/files/ censo2005/etnia/sys/colombia nacion.pdf. Consultado: 6 de marzo de 2011

3. Cultura y pervivencia del pueblo Yanacona en la ciudad. [Sitio en internet]. Disponible en: http://indigena.todosatierra. com/?p=206. Consultado: 13 de octubre de 2012.

4. Colleen W. La muerte de las lenguas indígenas: la pérdida de la diversidad. GHM. [En línea] 2003 [fecha de acceso 28 de julio de 2012]; 1-20 URL disponible en: http://cat.middlebury.edu/-gacetahispanica/trabajos/Lamuertedelaslenguasindig_ColleenWalsh.pdf

5. Organización mundial de la salud. Medicina tradicional. [Sitio en internet] disponible en: http://www.who.int/mediacentre/ factsheets/fs134/es/index.html. Consultado: 21 de julio de 2012.

6. Arinzon Z, Shabat S, Peisakh A, Berner Y. Clinical presentation of urinary tract infection (UTI) differs with aging in women. Archives of Gerontology and Geriatrics [en línea] 24 de julio del 2011 [fecha de acceso 4 de agosto de 2012]; 55 (2012) 145-147. URL disponible en: http://www.aggjournal. com/article/S0167-4943(11)00220-2/pdf

7. Rivero Z, Maldonado A, Bracho A, Gotera J, Atencio R, Leal $\mathrm{M}$ et. al. Enteroparasitosis en indígenas de la comunidad japrería, estado zulia, Venezuela. Interciencia [en línea] abril 2007 [fecha de acceso 29 de julio de 2012]; 32(4): 270-273. URL disponible en: http://redalyc.uaemex.mx/ pdf/339/33932411.pdf

8. Devera R, Finali M, Franceschi G, Gil S, Quintero O. Elevada prevalencia de parasitosis intestinales en indígenas del Estado Delta Amacuro, Venezuela. RevBiomed [en línea] octubre-diciembre 2005 [fecha de acceso 29 julio de 2012]; 16(4): 289291. URL disponible en: http://www.medigraphic.com/pdfs/ revbio/bio-2005/bio054i.pdf.

9. Vásquez A, Granados V, Franco D, Cárdenas C, Latorre J, Manascero A, et. al. Enfermedades parasitarias, factores de riesgo e inmunidad secretora en las comunidades indígenas y negras visitadas por la gran expedición humana. Un estudio piloto. Bogotá: terrenos; 1994. ( Serie de reportes de investigación: 3).

10. García M, Leets I, Bracho C, Hidalgo M, Bastidas G, Gómez A et. al. Prevalence of anemia and deficiencies of iron, folic acid and vitamin B12 in an Indigenous community from the Venezuelan Amazon with a high incidence of malaria.ALAN [en línea] 2008 [fecha de acceso 29 de julio de 2012]; 58(1): 12-18 URL disponible en: http://www.scielo.org.ve/pdf/alan/ v58n1/art02.pdf.

11. Leon Huerta, Bibiana M., NunezZarazu, Llerme y Alberto Veramendi, Verónica. Estado nutricional, anemia ferropénica y parasitosis intestinal en niños menores de cinco años 
del asentamiento humano de Chayhua distrito de Huaraz 2008. Aporte Santiaguino. [en línea]. Ene-jun 2009 [fecha de acceso 29 de julio de 2012] 2(1) p.159-163. URL Disponible en: <http://revistas.concytec.gob.pe/scielo. php? script=sci arttext\&pid=S2070-836X2009000100022\&l ng=es\&nrm=iso $>$. ISSN 2070-836.

12. Cook J. Diagnosis and management of iron-deficiencyanaemia. [en línea] 2005 [fecha de acceso 24 de agosto de 2012] 18 (2). pp. 319-332. URL Disponible en: http://ac.els-cdn.com. biblioteca.uniandes.edu.co:8080/S152169260400088X/1s2.0-S152169260400088X-main.pdf? tid=f402507a-f18c$11 \mathrm{e} 1-808 \mathrm{~d}-00000 \mathrm{aab} 0 \mathrm{f} 27$ \&acdnat $=1346212529 \quad 873 \mathrm{ec} 39 \mathrm{fe}$ 6ab2291179725645afc2b78.

13. Organización Mundial de la Salud. Assessing the iron status of populations: including literature reviews.Report of Joint World Health Organization/Centers for Disease Control and Prevention Technical Consultation on the Assessment of Iron
Status at the Population Level.[ Sitio en internet]. Disponible en: http://www.who.int/nutrition/publications/micronutrients/anaemia iron deficiency/9789241596107.pdf. Consultado: 18 de agosto de 2012.

14. García M, Leets I, Bracho C, Hidalgo M, Bastidas G, Gómez A et. al. Prevalence of anemia and deficiencies of iron, folic acid and vitamin B12 in an Indigenous community from the Venezuelan Amazon with a high incidence of malaria.ALAN [en línea] 2008 [fecha de acceso 29 de julio de 2012]; 58(1): 12-18 URL disponible en: http://www.scielo.org.ve/pdf/alan/v58n1/ art02.pdf.

15. 15. Grange E, Semont K, Meknache N, Giraudeax V, Chappuis P. Las disferritinemias, algoritmo de orientación diagnóstica. Acta bioquím. clín. latinoam. [en línea] junio del 2006 [fecha de acceso 17 de agosto de 2012]; 40 (2). Disponible en: http://www.scielo.org.ar/scielo.php?script=sci arttext\&pi $\mathrm{d}=\mathrm{S} 0325-29572006000200016$ 